\title{
High expression of ubiquitin-conjugating enzyme 2C (UBE2C) correlates with nasopharyngeal carcinoma progression
}

\author{
Zhihua Shen ${ }^{1+}$, Xiaofan Jiang ${ }^{1+}$, Chao Zeng ${ }^{1}$, Shaojiang Zheng ${ }^{2}$, Botao Luo ${ }^{1}$, Yumei Zeng ${ }^{3}$, Ranran Ding ${ }^{1}$,
} Hanguo Jiang ${ }^{1}$, Qiyi He${ }^{2}$, Junli Guo ${ }^{2^{*}}$ and Wei Jie 1* $^{*}$

\begin{abstract}
Background: Overexpression of ubiquitin-conjugating enzyme 2C (UBE2C) has been detected in many types of human cancers, and is correlated with tumor malignancy. However, the role of UBE2C in human nasopharyngeal carcinoma (NPC) is unclear. In this study, we investigated the role of aberrant UBE2C expression in the progression of human NPC.

Methods: Immunohistochemical analysis was performed to detect UBE2C protein in clinical samples of NPC and benign nasopharyngeal tissues, and the association of UBE2C expression with patient clinicopathological characteristics was analyzed. UBEC2 expression profiles were evaluated in cell lines representing varying differentiated stages of NPC and immortalized nasopharyngeal epithelia NP-69 cells using quantitative RT-PCR, western blotting and fluorescent staining. Furthermore, UBE2C was knocked down using RNA interference in these cell lines and proliferation and cell cycle distribution was investigated.
\end{abstract}

Results: Immunohistochemical analysis revealed that UBE2C protein expression levels were higher in NPC tissues than in benign nasopharyngeal tissues $(P<0.001)$. Moreover, high UBE2C protein expression was positively correlated with tumor size $(P=0.017)$, lymph node metastasis $(P=0.016)$ and distant metastasis $(P=0.015)$ in NPC patients. In vitro experiments demonstrated that UBE2C expression levels were inversely correlated with the degree of differentiation of NPC cell lines, whereas UBE2C displayed low level of expression in NP-69 cells. Knockdown of UBE2C led to significant arrest at the $S$ and G2/M phases of the cell cycle, and decreased cell proliferation was observed in poorly-differentiated CNE2Z NPC cells and undifferentiated C666-1 cells, but not in well-differentiated CNE1 and immortalized NP-69 cells.

Conclusions: Our findings suggest that high expression of UBE2C in human NPC is closely related to tumor malignancy, and may be a potential marker for NPC progression.

Keywords: Nasopharyngeal carcinoma, Ubiquitin-conjugating enzyme 2C, Progression, Proliferation, Cell cycle

\section{Background}

Ubiquitination is a crucial molecular mechanism for the degradation of short-lived proteins in eukaryotic cells, and is involved in multiple cellular biological processes including the cell cycle. The process of protein monoubiquitination or polyubiquitination occurs under the

\footnotetext{
*Correspondence: maomao0511051@yahoo.com.cn; Jiewei74@126.com ${ }^{\dagger}$ Equal contributors

${ }^{2}$ Hainan Provincial Key Laboratory of Tropical Medicine, Hainan Medical College, Haikou 571199, People's Republic of China

'Department of Pathology \& Pathophysiology, School of Basic Medicine Science, Guangdong Medical College, Zhanjiang 524023, People's Republic of China

Full list of author information is available at the end of the article
}

control of three types of enzymes: E1 ubiquitin-activating enzymes, E2 ubiquitin-conjugating enzymes and E3 ubiquitin ligase [1]. Human ubiquitin-conjugating enzyme E2C (UBE2C, also called UBCH10) encodes a member of the E2 ubiquitin-conjugating enzyme family [2]. It was reported that UBE2C functions closely with the anaphasepromoting complex/cyclosome (APC/C), which is an E3 ubiquitin ligase that targets cell cycle proteins for degradation by the proteasome [3]. UBE2C is required for the destruction of mitotic cyclins, thereby participating in the regulation of cell cycle progression through $\mathrm{M}$ phase [2].

In 2003, Okamoto et al. demonstrated that UBE2C expression levels were extremely low in many normal

\section{Biomed Central}


tissues, but prominent in the majority of cancerous cell lines examined, suggesting that UBE2C has the ability to promote cell proliferation and malignant transformation [4]. Recent data has shown that aberrantly high expression of UBE2C contributes to tumorigenesis, and has revealed its potential as a biomarker for cancer prognosis [5]. Abnormally high UBE2C expression was observed in various human solid cancers in the liver [6], thyroid [7], breast [8], colon [9,10], cervix [11], lung [12] and brain [13], and UBE2C expression was positively correlated with invasion depth and tumor node metastasis (TNM) stage in some tumors. Furthermore, inhibition of UBE2C expression induced by RNA interference significantly reduced the proliferation of cancer cells [7,14] and enhanced cell apoptosis in vitro [15]. UBE2C transgenic mice are prone to carcinogen-induced lung tumors and a broad spectrum of spontaneous tumors, as UBE2C is a prominent proto-oncogene [16]. Taken together, these data suggest that targeting of UBE2C may be a potential tool for tumor diagnosis and therapy.

Nasopharyngeal carcinoma (NPC) is a type of malignant head and neck cancer derived from the nasopharyngeal epithelium, and is one of the most common malignant diseases in Southern China and Southeast Asia [17]. Almost 85\% of NPC patients display a more advanced clinical stage of disease because of the prevalence of lymphadenopathy at first diagnosis [18]. The process of NPC formation and metastasis is complex, and various genes are involved [19] Therefore, it is of great importance to research biomarkers for the early diagnosis, prognosis prediction of NPC and to develop novel therapeutic strategies for NPC. In the present study, we aimed to investigate the role of UBE2C in the progression of NPC. Our results indicated that detection and targeting of UBE2C may be a potentially useful biomarker for NPC treatment.

\section{Methods}

\section{Patient samples}

One hundred and fifteen cases of paraffin-embedded clinical samples were obtained from the Affiliated Hospital of the Guangdong Medical College (Zhanjiang City, Guangdong, China) and the People's Hospital of Zhongshan City (Zhongshan City, Guangdong, China). In total, 91 cases of NPC $(n=91)$ and 24 cases of nasopharyngeal epithelial hyperplasia $(\mathrm{NEH})$ were examined from 69 men $(75.8 \%)$ and 22 women $(24.2 \%)$. Clinical stage was classified based on the pathology tumornode-metastasis (pTNM) system (AJCCUICC 2002), and all NPC samples were determined to be nonkeratinizing carcinoma. NPC patients were diagnosed for the first time at an average age of 42.7 years (range, 2372 years). Additional clinical data are shown in Table 1. The use of human tissues in this study was approved by the
Table 1 Clinicopathological characteristics of patient samples and UBE2C expression in NPC

\begin{tabular}{lc}
\hline & $\mathrm{N}(\%)$ \\
\hline Gender & \\
Male & $69(75.8)$ \\
Female & $22(24.2)$ \\
Age & \\
\50 & $44(48.4)$ \\
$<50$ & $47(51.6)$ \\
Smoking & \\
Yes & $4246.2)$ \\
No & $49(53.8)$ \\
Clinical classification & \\
I-II & $15(16.5)$ \\
III-IV & $76(83.5)$ \\
T classification & \\
T1-T2 & $31(34.1)$ \\
T3-T4 & $60(65.9)$ \\
N classification & \\
N0 & $19(20.9)$ \\
N1-N3 & $7279.1)$ \\
M classification & \\
M0 & \\
M1 & $77(84.6)$ \\
Expression of UBE2C & $14(15.4)$ \\
High expression & \\
Low expression & $40(44.0)$ \\
\hline & \\
\hline & \\
\hline
\end{tabular}

Ethics Council of the Affiliated Hospital of the Guangdong Medical College and the People's Hospital of Zhongshan City for Approval of Research Involving Human Subjects.

\section{Immunohistochemical analysis of UBE2C protein}

The expression and cellular distribution of UBE2C protein was assessed by immunohistochemical analysis. Five micrometer-thick paraffin sections were deparaffinized and re-hydrated according to standard protocols, and heat-induced antigen retrieval was performed in sodium citrate buffer $(10 \mathrm{mmol} / \mathrm{L}, \mathrm{pH} 6.0)$. Endogenous peroxidase was inhibited by $0.3 \% \mathrm{H}_{2} \mathrm{O}_{2}$, and non-specific protein binding was blocked with $10 \%$ goat serum. Sections were then incubated with primary antibody against UBE2C (1:200 dilution; cat. \#A-650, Boston Biochem, MA, USA) at $4^{\circ} \mathrm{C}$ overnight. Non-immune IgG was used as a negative control, and antigenic sites were localized using a SP9000 Polymer Detection System and a 3,3'diaminobenzidine (DAB) kit (ZSGB-BIO, Beijing, China). The immunoreactive score (IRS) of UBE2C was described previously [20]. Briefly, the staining intensity was 
determined as 0 , negative; 1 , weak; 2 , moderate; and 3, strong. The percentage of UBE2C-positive cells was scored as 0 , no cellular staining; $1,<1 \%$ cellular staining; 2, $1-10 \%$ cellular staining; $3,10-33 \%$ cellular staining; 4 , $33-66 \%$ cellular staining; and $5,>66 \%$ cellular staining. Samples with a total IRS of $<6$ were deemed as having low UBE2C expression, and samples with a sum IRS of $\geq 6$ were determined as high UBE2C expression. The scoring of $\mathrm{UBE} 2 \mathrm{C}$ was evaluated individually and independently by two pathologists who were double-blinded to the clinical data.

\section{Cell culture}

CNE1, CNE2Z and C666-1 cell lines representing well-, poorly- and undifferentiated NPC, respectively, were grown in Dulbecco's modified Eagle's medium (DMEM; Hyclone) supplemented with 10\% fetal bovine serum (FBS; Hyclone) and $100 \mathrm{U} / \mathrm{ml}$ penicillin and streptomycin $(100 \mu \mathrm{g} / \mathrm{ml})$, as described previously [21]. The immortalized nasopharyngeal epithelial cell line NP-69 (obtained from the lab of Prof. Yao K.T., Cancer Research Institute, Southern Medical University, Guangzhou, China) was cultured in defined keratinocyte serum-free medium (cat. \#10744-019, Invitrogen) containing $100 \mathrm{U} / \mathrm{ml}$ penicillin, $100 \mu \mathrm{g} / \mathrm{ml}$ streptomycin, $0.2 \mathrm{ng} / \mathrm{ml}$ recombinant epidermal growth factor and 5\% FBS. All cell lines were cultured at $37^{\circ} \mathrm{C}$ in a humidified atmosphere with $5 \% \mathrm{CO}_{2}$.

\section{RNA interference}

siRNAs were purchased from RiboBio Co., Ltd. (Guangzhou, China). For RNA interference (RNAi) experiments, the following double-stranded oligo RNAs specific for the $U B E 2 C$ coding region (si-UBE2C) were used: forward, 5' -GGACACCCAGGGUAACAUAdTdT-3', reverse, 5'-UAUGUUACCCUGGGUGUCCdTdT-3'. A corresponding scrambled sequence (si-Control, Cat. siB05815) was used as a negative control. One day before transfection, equal numbers of CNE1, CNE2Z, C666-1 and NP-69 cells $\left(5.0 \times 10^{5} / \mathrm{ml}\right)$ were seeded in 6-, 24- and 96-well plates supplemented with complete medium without antibodies. When cells had reached $60-70 \%$ confluency, they were transfected with siRNAs using Lipofectamine 2000 (Invitrogen) in Opti-MEM I medium (Invitrogen). Cells were incubated at $37^{\circ} \mathrm{C}$ in a humidified atmosphere of $5 \%$ $\mathrm{CO}_{2}$ for $6 \mathrm{~h}$ followed by replacement of complete medium. The efficiency of transfection was verified by observation of the fluorescence emitted by the Cy3-conjugated si-Control using fluorescence microscopy.

\section{Immunofluorescent staining}

Indirect immunofluorescence was performed on NPC cells cultured on glass coverslips. After overnight incubation with primary antibody against UBE2C $(1 / 100)$ at $4^{\circ} \mathrm{C}$, the antigenic sites were detected using TRITC-conjugated goat anti-rabbit IgG (1/100, Protein Tech Group, Inc., Chicago, IL, USA). Images of the antigenic sites were captured with a laser scanning confocal microscope (TCS SP5 II; Leica, Germany).

\section{Western blotting}

Total proteins were extracted using RIPA lysis buffer (Cat. \# P0013C, Beyotime Institute of Biotechnology, Jiangsu, China). $30 \mu \mathrm{g}$ total proteins were subjected to SDS-PAGE, and then proteins were transferred to the PVDF membranes. After twice washed with TBST, the membranes were incubated with $5 \%$ skimmed milk in TBST at $37^{\circ} \mathrm{C}$ for $30 \mathrm{~min}$, then the membrane were incubated with the primary antibodies (UBE2C, 1:500, Boston Biochem; $\beta$-actin, 1:1000, Santa Cruz, Texas, USA) at $4^{\circ} \mathrm{C}$ overnight, After twice washed by TBST, the membranes were incubated with horseradish peroxidase (HRP)-conjugated secondary antibodies for 1 hour at $37^{\circ} \mathrm{C}$. Bands were visualized using enhanced chemiluminescence (ECL) reagents (Thermo Fisher, Rockford, IL, USA) and analyzed with gel analysis system (BIO-RAD VersDoc TM5000MP System, Guangzhou, China). The expression of $\beta$-actin was used as loading control.

\section{RNA extraction and quantitative RT-PCR}

Total RNA was extracted with TaKaRa RNAiso plus reagent (Takara Biotechnology (Dalian) Co., Ltd.). Next, $1 \mu \mathrm{g}$ of total RNA was used as a template to generate the first strand cDNA by oligo $\left(\mathrm{dT}_{18}\right)$ using the Promega RT System. Pairs of primers $\left(5^{\prime}-3^{\prime}\right)$ synthesized by Sangon Biotech Co., Ltd. (Shanghai, China) were as follows: $U B E 2 C$ forward: tgatgtctggcgataaagggatt, $U B E 2 C$ reverse: gtgatagcagggcgtgaggaa. $\beta$-actin forward, tgacgtggacatccgc aaag, $\beta$-actin reverse, ctggaaggtggacagcgagg. PCR was conducted using the LightCycler480 II instrument (Roche (China) Ltd., Shanghai, China). The total reaction volume of $10 \mu \mathrm{l}$ consisted of $5 \mu \mathrm{l} \mathrm{SYBR}$ Green I PCR Master Mix (Toyobo, Osaka, Japan), $0.4 \mu \mathrm{l}$ forward primer $(10 \mu \mathrm{M})$, $0.4 \mu \mathrm{l}$ reverse primer $(10 \mu \mathrm{M}), 1 \mu \mathrm{l}$ cDNA and $3.2 \mu \mathrm{l}$ $\mathrm{ddH}_{2} \mathrm{O}$. The PCR amplification protocol was as follows: denaturation was performed at $95^{\circ} \mathrm{C}$ for $1 \mathrm{~min}$, followed by $45 \mathrm{PCR}$ cycles of $95^{\circ} \mathrm{C}$ for $15 \mathrm{~s}$, and $60^{\circ} \mathrm{C}$ for $60 \mathrm{~s}$. The relative abundance of target mRNAs were determined from the $C_{\mathrm{T}}$ values and plotted as the fold change compared with the control group.

\section{In vitro proliferation assays}

Proliferation rates were determined by Cell Counting Kit- 8 (CCK-8) assays, as described previously [21]. Briefly, $4 \times 10^{3}$ cells were seeded in 96-well plates at either 24 and $48 \mathrm{~h}$ after transfection with or without siRNAs, then $10 \mu \mathrm{l} \mathrm{CCK-}$ 8 reagent (Beyotime Institute of Biotechnology, Jiangsu, China) plus $100 \mu \mathrm{l}$ basal DMEM medium was added per well, and the absorbance of the samples was measured. Each independent experiment was performed three times. 


\section{Cell cycle distribution analysis}

NPC cell lines were seeded in 6-well plates and were successfully transfected in triplicate for each set of experimental conditions with the siRNAs described above. Forty-eight hours later, harvested cells were stained with propidium iodide (PI) and subjected to flow cytometric analysis (BD FACSCanto II, MA, USA).

\section{Statistical analyses}

Statistical analyses were carried out using PRISM Software (Version 5. GraphPad Software, CA, USA). Data were analyzed with Chi-square tests and expressed as mean \pm SD. For analysis of the differences between two groups, Student's t-tests were performed. For multiple groups, ANOVA was carried out followed by StudentNewman-Keuls tests. The level of statistical significance was set at $P<0.05$.

\section{Results}

Immunohistochemical analysis of UBE2C protein expression in NPC and nasopharyngeal tissues First, we investigated the expression of UBE2C in NEH and NPC. Immunohistochemical staining revealed that the majority of NEH cases displayed no or low levels of UBE2C protein expression (IRS <6, 24/24); however, $56 \%$ of NPCs (51/91) exhibited strong nuclear and cytoplasmic UBE2C immunoreactivity (IRS $\geq 6)(P<0.001$ when compared with
$\mathrm{NEH}$ ), indicating a crucial role of UBE2C expression in the pathogenesis of NPC (Table 1 and Figure 1).

\section{Relationship between clinicopathological characteristics and UBE2C protein expression in NPC patients}

The relationships between clinicopathological parameters and UBE2C protein expression levels in NPCs are detailed in Table 2. There was no significant association of high UBE2C protein expression levels with age, sex, smoking and clinical stage (I-II vs. III-IV) in 91 NPC cases. However, we observed that the level of UBE2C protein expression was positively correlated with tumor size (T classification) (T1-T2 vs. T3-T4, $P=0.017)$, lymph node metastasis (N classification) (N0 vs. N1-N3) $(P=0.016)$ and distant metastasis (M classification) (M0 vs. M1, $P=0.015$ ) in NPC patients (Table 2). These data indicated that UBE2C overexpression may be associated with the clinical progression of NPC.

\section{Expression profiles of UBE2C in NPC cell lines in vitro} CNE1, CNE-2Z and C666-1 cells were used to further examine the expression profiles of UBE2C in NPC cell lines in the present study. As shown in Figure 2, variable expression of UBE2C was observed at both the mRNA and protein levels in various NPC cell lines. In general, lower expression of UBE2C was detected in highly differentiated CNE1 cells, while increasing expression levels
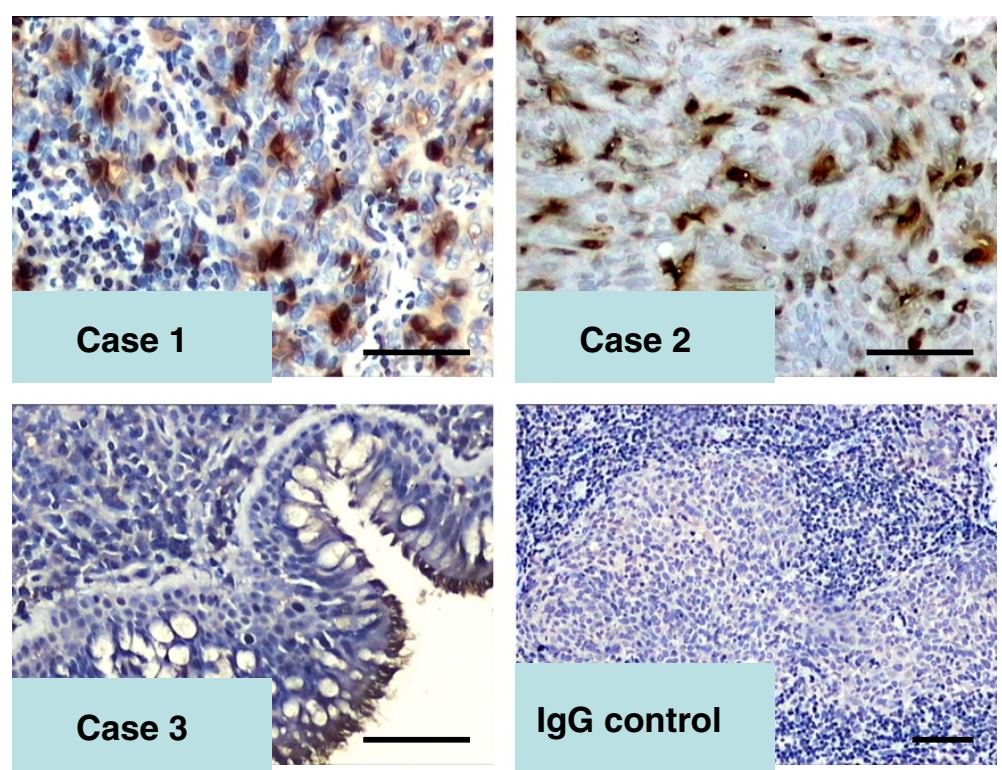

Figure 1 Representative photographs of high UBE2C expression in NPC samples and low UBE2C expression in non-cancerous nasopharyngeal epithelial hyperplasia (NEH). The immunohistochemical PV9000 method was used to detect UBE2C protein expression in clinical samples. Non-immune lgG was used as a negative control. The expression and location of UBE2C in cells was revealed by staining with DAB and counterstaining with hematoxylin. IRS $>6.0$ in NPC (cases 1 \& 2) and IRS $<6.0$ in NEH (case 3). Original magnification for cases 1-3, $\times 200$, for the lgG control, $\times 100$. Scale bar $=100 \mu \mathrm{M}$. 
Table 2 Correlation between clinicopathological characteristics and UBE2C protein expression in NPC

\begin{tabular}{|c|c|c|c|c|c|}
\hline \multirow{2}{*}{ Clinical parameters } & \multirow{2}{*}{$\mathrm{n}$} & \multicolumn{2}{|c|}{ UBE2C expression } & \multirow{2}{*}{$x^{2}$} & \multirow{2}{*}{$P$-value } \\
\hline & & high & low & & \\
\hline \multicolumn{6}{|l|}{ Histological types } \\
\hline NPC & 91 & 51 & 40 & \multirow{2}{*}{26.80} & \multirow{2}{*}{$0.0001^{*}$} \\
\hline NEH & 24 & 0 & 24 & & \\
\hline \multicolumn{6}{|l|}{ Smoking } \\
\hline Yes & 42 & 24 & 18 & \multirow{2}{*}{0.038} & \multirow{2}{*}{0.845} \\
\hline No & 49 & 27 & 22 & & \\
\hline \multicolumn{6}{|l|}{ Gender } \\
\hline Male & 69 & 37 & 32 & \multirow{2}{*}{0.679} & \multirow{2}{*}{0.410} \\
\hline Female & 22 & 14 & 8 & & \\
\hline \multicolumn{6}{|l|}{ Age } \\
\hline$\geqq 50$ & 44 & 22 & 22 & \multirow{2}{*}{1.263} & \multirow{2}{*}{0.261} \\
\hline$<50$ & 47 & 29 & 18 & & \\
\hline \multicolumn{6}{|l|}{ Clinical classification } \\
\hline I-II & 15 & 6 & 9 & \multirow{2}{*}{1.877} & \multirow{2}{*}{0.171} \\
\hline III-IV & 76 & 45 & 31 & & \\
\hline \multicolumn{6}{|l|}{ T classification } \\
\hline $\mathrm{T} 1-\mathrm{T} 2$ & 31 & 12 & 19 & \multirow{2}{*}{5.735} & \multirow{2}{*}{$0.017^{*}$} \\
\hline T3-T4 & 60 & 39 & 21 & & \\
\hline \multicolumn{6}{|l|}{$\mathrm{N}$ classification } \\
\hline No & 19 & 6 & 13 & \multirow{2}{*}{5.835} & \multirow{2}{*}{$0.016^{*}$} \\
\hline N1-N3 & 72 & 45 & 27 & & \\
\hline \multicolumn{6}{|l|}{ M classification } \\
\hline Mo & 77 & 39 & 38 & \multirow{2}{*}{5.913} & \multirow{2}{*}{$0.015^{*}$} \\
\hline M1 & 14 & 12 & 2 & & \\
\hline
\end{tabular}

* Significance as indicated.

of UBE2C were observed in CNE2Z cells (poorly differentiated NPC) and C666-1 cells (undifferentiated NPC). Low level of UBE2C expression was also observed in immortalized NP-69 cells (Figures 2 and 3). These results indicated that UBE2C was universally expressed in the NPC cell lines, and its expression levels were inversely associated with differentiation status. Finally, immunofluorescent staining showed that UBE2C protein was cytoplasmic in immortalized NP-69 cells, but localized to the cytoplasm and nuclei of NPC cell lines (Figure 3).

\section{Knockdown of UBE2C attenuates NPC proliferation}

Forced UBE2C expression in NIH 3T3 cells has been shown to promote cell proliferation [4]. Thus, we examined the role of UBE2C in NPC cell proliferation. Three pairs of RNA oligos targeting different regions of the $U B E 2 C$ gene coding region were designed to knockdown UBE2C expression (data not shown). We found that the

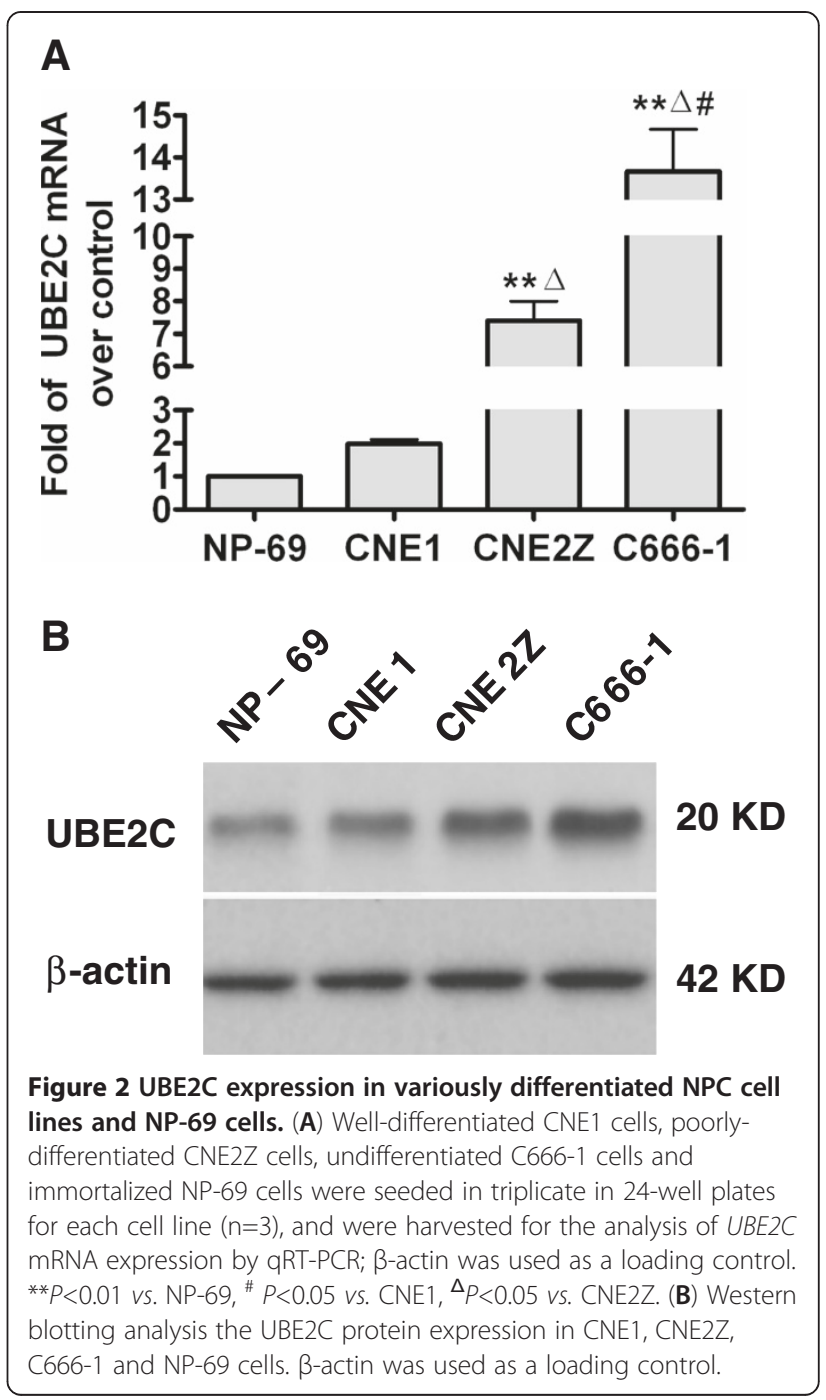

double-stranded oligos targeting the sequence GGACACC CAGGGTAACATA (401-420nt of CDS of UBE2C) displayed the most powerful inhibitory effects $(>75 \%)$. As shown in Figure 4A, si-UBE2C attenuated UBE2C expression both at the mRNA and protein levels in high UBE2Cexpressing C666-1 cells, indicating these siRNA oligos function well. Therefore, these double-stranded RNA oligos were used in the subsequent experiments. And the results of western blotting further confirmed that transfection this siRNAs to NPC cells led to a significant decrease of UBE2C protein expression (Figure 4B). Then the cell proliferation was examined by CCK- 8 assays post transfection these 4 cell line with UBE2C specific siRNA. As shown in Figure 4C, transfection of the NPC cell lines with this siRNA led to significantly damaged cell viability in CNE2Z and C666-1 cells, but not the CNE1 and NP-69 cells. Together, these results suggest that overexpression of UBE2C plays a crucial role in NPC cell proliferation. 


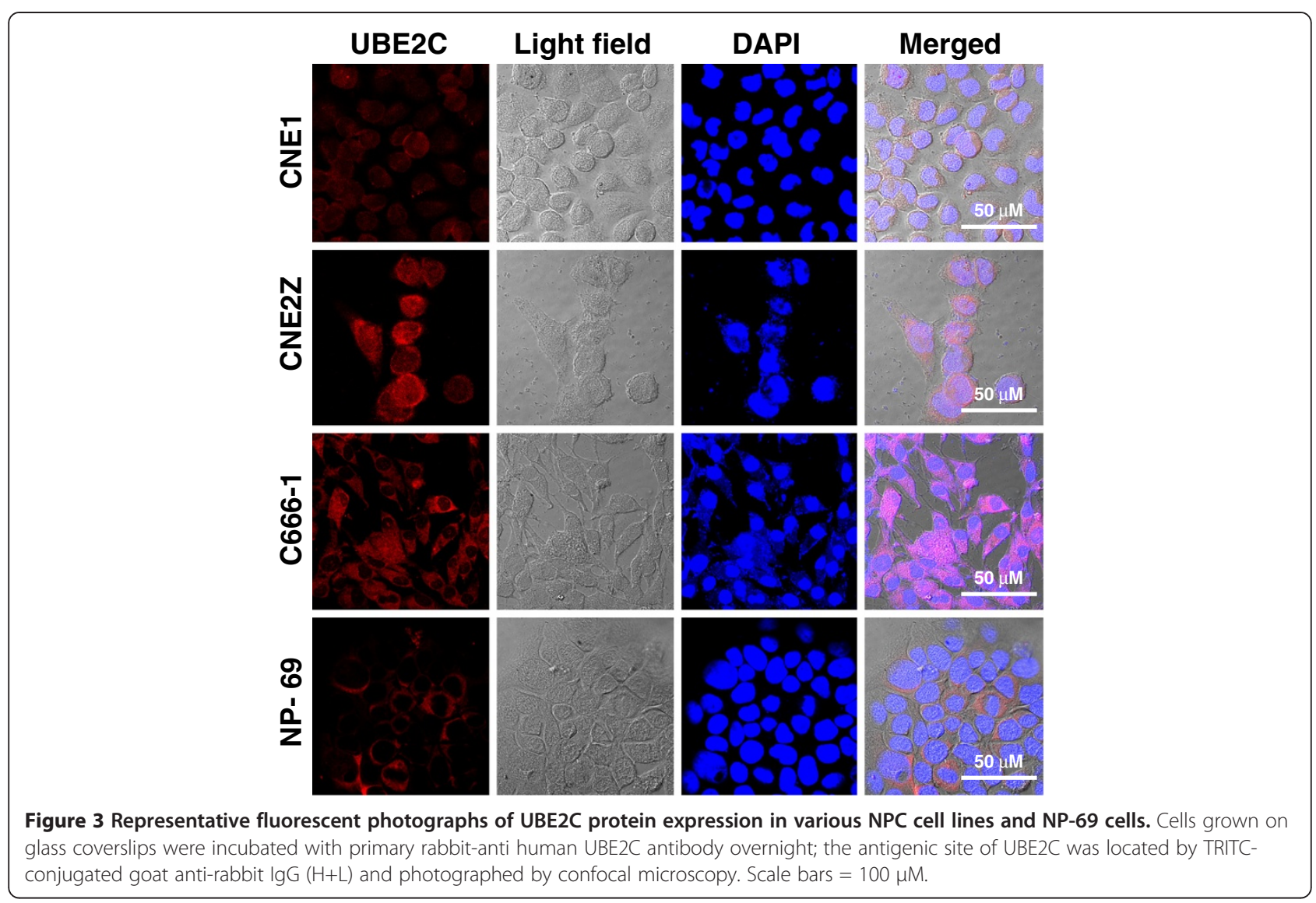

\section{Knockdown of UBE2C arrests NPC cells at S and G2/M phases}

UBE2C is involved in many points of cell cycle control [5]. In the present study, treatment of the NPC cell lines with si-UBE2C decreased the distribution of cells in G1 phase but increased the proportion in $\mathrm{S}$ and $\mathrm{G} 2 / \mathrm{M}$ phase. As shown in Figure 5, the increases in the proportion of NP-69, CNE1, CNE2Z and C666-1 cells in S phase was $35.7 \%, 30.9 \%, 79.9 \%$ and $141.6 \%$, respectively. Furthermore, the increase in the proportion of NP-69, CNE1, CNE2Z and C666-1 cells in G2/M phase was $26.4 \%, 21.1 \% 92.8 \%$ and $110.3 \%$, respectively. These results suggested that inhibition of UBE2C expression in UBE2C highly-expressing NPC cells led to a significant re-distribution in the cell cycle.

\section{Discussion}

In the present study, we first found that UBE2C was predominantly expressed in NPC samples, whereas it was weakly expressed in nasopharyngeal tissues; moreover, we found that high UBE2C protein expression was positively related to tumor size, lymph node metastasis and distant metastasis in NPC patients. These results indicated that high expression of UBE2C was closely related to the clinical progression of NPC. Consequently, we examined
UBE2C expression in variously differentiated NPC cell lines in vitro. The results showed that immortalized nasopharyngeal NP-69 cells displayed low level of UBE2C expression; however, UBE2C was universally expressed in a variety of NPC cell lines, and its expression levels were reversely related to the stages of differentiation. Finally, treatment of the NPC cells with UBE2C-specific siRNA led to a decrease in cell proliferation and arrest at $\mathrm{S}$ and G2/M phase of the cell cycle, suggesting that targeting of UBE2C is a potential anti-NPC therapeutic strategy. To the best of our knowledge, this is the first report regarding the relation of aberrant expression of UBE2C with NPC malignancy.

Human UBE2C belongs to the E2 ubiquitin-conjugating enzyme family [2], which functions closely with APC/C [3]. Expression of UBE2C is required for the destruction of mitotic cyclins, for example cyclin B, to promote cell cycle progression from $M$ to $G 1$ phase [2]. Therefore, overexpression of UBE2C contributes to increased cell proliferation, and as a result, cancer cells acquire a hallmark of tumorigenicity through uncontrolled cell proliferation. Early work by Fang et al. revealed that some candidate biomarkers for cancer, including UBE2C, were upregulated in NPC [22]. In the present study, we found that high expression of UBE2C protein was detected in 

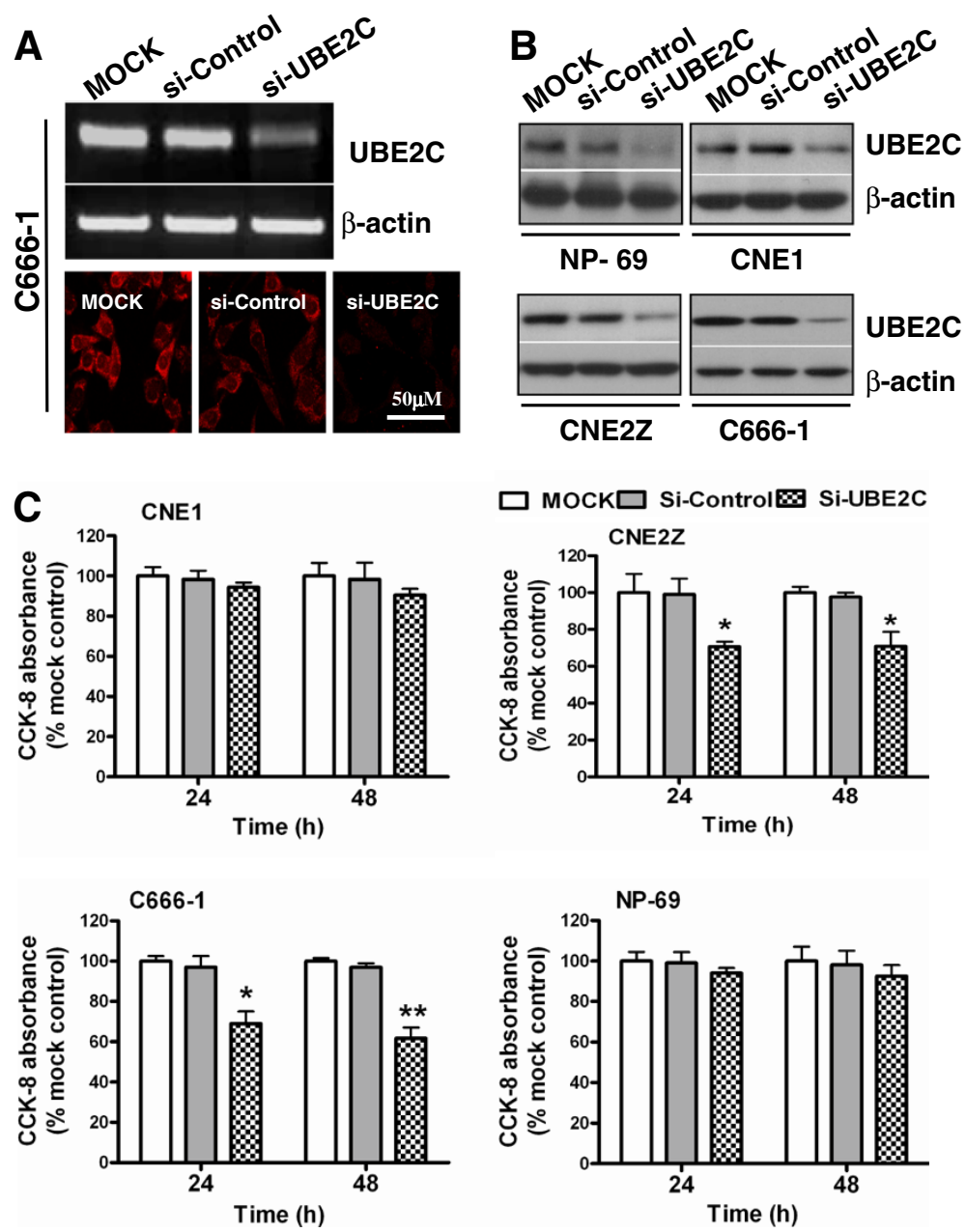

Figure 4 siRNA inhibited UBE2C expression in NPC cells and consequently resulted in attenuated cell proliferation. (A) C666-1 cells were transfected with si-UBE2C or si-Control or without siRNA (MOCK). Forty-eight hours later, UBE2C expression was assessed by PCR and immunofluorescence using TRITC-conjugated $\operatorname{lgG}(\mathrm{H}+\mathrm{L})$. (B) Western blotting analysis the UBE2C protein expression in various cell lines post tranfection of siRNAs, the $\beta$-actin was used as loading controls. $(\mathbf{C})$ Twenty-four and $48 \mathrm{~h}$ after transfection, CCK-8 assays were used to analyze the proliferation of various types of NPC cells. Values of optical density (OD) were obtained by the absorbance at the dual wavelengths $450 / 630 \mathrm{nM}$, and the results indicating the cell viability were plotted as the percentage over controls (MOCK cells). ${ }^{*} P<0.05,{ }^{* *} P<0.01$ vs. mock or si-Control-treated groups.

56.0\% NPC cases, whilst no UBE2C expression was observed in benign nasopharyngeal tissues; moreover, high UBE2C expression was found to be positively associated with the T, $\mathrm{M}$ and $\mathrm{N}$ classifications of NPC, indicating that high expression of UBE2C contributes to the pathogenesis and clinical progression of NPC, although these findings require further validation in larger cohorts. Our results were consistent with other reports describing overexpression of UBE2C in many types of tumors, and demonstrate that detection of UBE2C may be a potential biomarker for tumor diagnosis or prognostic judgment [6-9,13,20,23-29].

By using a variety of differentiated stages of NPC cell lines, the UBE2C expression profiles were further analyzed. Well-differentiated CNE1, poorly-differentiated CNE2Z and undifferentiated C666-1 cells used in the present investigation were representative of NPC. We found that when compared with the immortalized NP-69 cells, UBE2C mRNA and protein were universally expressed in these NPC cell lines. Generally, UBE2C expression was found to be inversely related with the differentiation stages of NPC cells. Poor differentiation in cancer cells implies a higher degree of malignancy, and as a hallmark of tumorigenesis, upregulated cell proliferation and migration was acquired. As a result, after treatment of the NPC cell lines with UBE2C-specific siRNA, attenuated cell proliferation was observed. Our results revealed that targeting UBE2C in NPC cells may be beneficial for NPC molecular treatment. These in vitro results were also consistent with other 

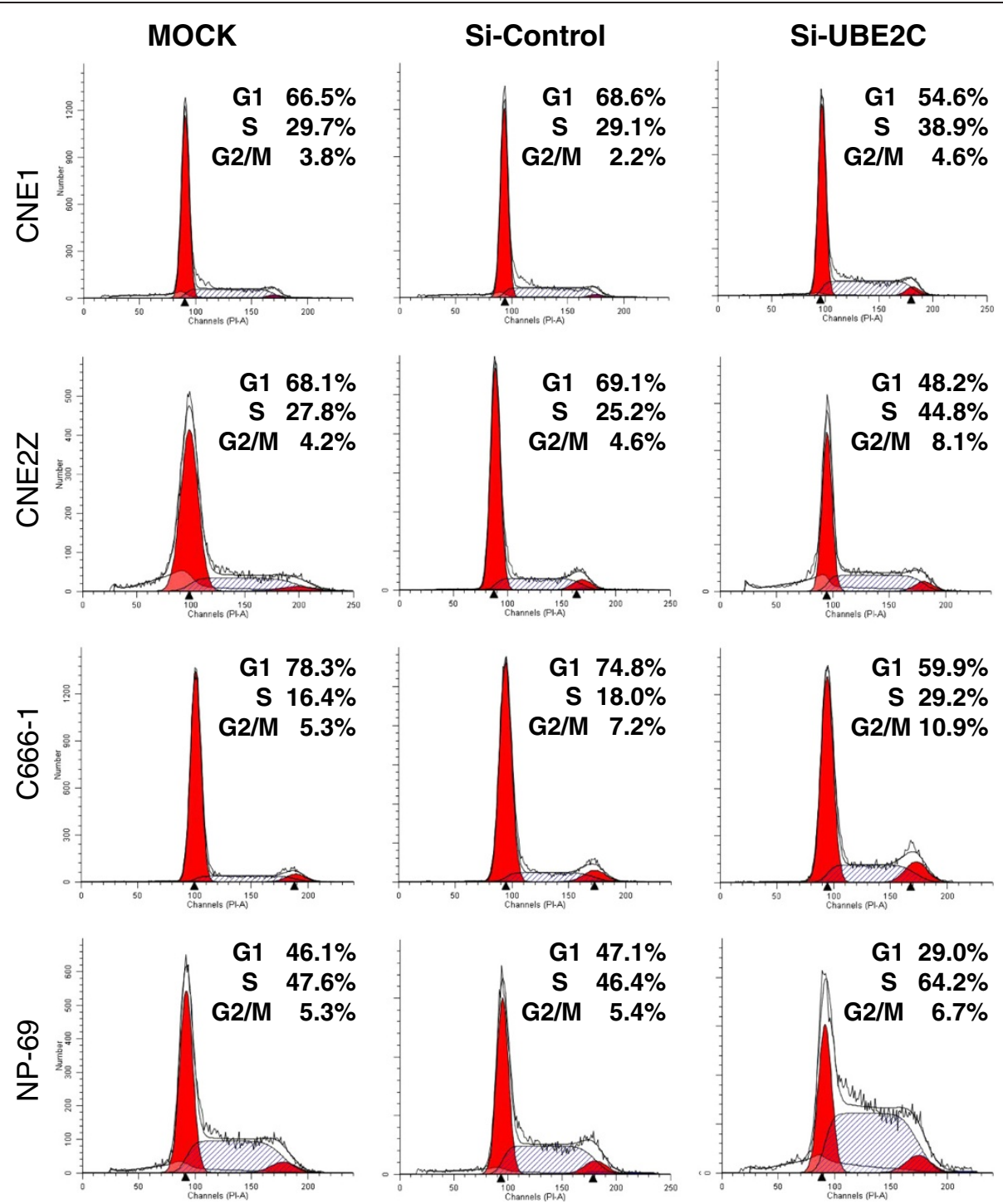

Figure 5 Knockdown of UBE2C with siRNA arrested the cell cycle at G2-M and S phase. CNE1, CNE2Z, C666-1 and NP-69 cells were transfected with UBE2C siRNA (si-UBE2C) or si-Control or left untransfected (MOCK) for $48 \mathrm{~h}$, then the cell cycle was assessed by FCM.

reports that targeting UBE2C may be a useful therapeutic strategy in various cancers, such as cervical, colorectal and esophageal carcinomas [11,14,25,30,31].

Cell cycle progression is precisely mediated by a combination of cyclin-dependent kinases, kinase inhibitors and protein phosphorylation. The timely and specific degradation of cyclins and kinase inhibitors at critical check points in the cell cycle by the ubiquitin-proteasome system (UPS) also participates in this process. The cell-cycle $\mathrm{G}_{2}-\mathrm{M}$ phase gene $U B E 2 C$ encompasses the cell cycle window associated with exit from mitosis. Depletion of UBE2C in cancer cells by $U B E 2 C$-siRNA redistributes the cell cycle phases $[14,25]$, while bortezomib or cell-cycle inhibitor-779 (CCI-779) stabilizes mitotic cyclins and prevents cell cycle progression via attenuation of UBE2C transcription and
mRNA stability [30,32]. Our present results revealed that knockdown of $U B E 2 C$ in NPC cells caused significant cellcycle G2-M and S accumulation. As our results show, transfection of the most highly UBE2C-expressing C666-1 cells with siRNA for $48 \mathrm{~h}$ lead to a $141.6 \%$ increase in G2$\mathrm{M}$ and $110.3 \%$ increase in $\mathrm{S}$ phase, implying a crucial role of UBE2C in NPC cell cycle determination. Our results support the findings of Lin et al., who reported that inhibition of UBE2C in Seg-1 cells with si-UBE2C resulted in the re-distribution of the cell cycle [25].

The UBE2C gene is localized to $20 \mathrm{q} 13.1$, a chromosomal region frequently associated with genomic amplification in many types of cancers. It was reported that genomic amplification was a mechanism of increased UBE2C expression in colon cancer, thyroid carcinoma and prostate 
cancer [23,33,34]. Extensive chromosomal copy number aberrations were also observed in NPC [35,36]. High frequencies of allelic imbalances at chromosomes $3 \mathrm{p}, 9 \mathrm{p}$, $11 \mathrm{q}, 12 \mathrm{q}, 13 \mathrm{q}, 14 \mathrm{q}$, and $16 \mathrm{q}$ were detected in primary NPC [37]. Very recently, Hu et al. reported a series of chromosomal abnormalities, including some of those hot spots mentioned above, in C666-1 cells and NPC biopsies [38]. In contrast to the previous investigations regarding amplification of 20q in some human tumors [23,33,34], the loss of 20q in NPC was reported by Yan et al. [39]. We did not examine the amplification of $20 \mathrm{q}$ in the present study; thus, the mechanism of high expression of UBE2C in NPC requires further elucidation.

NPC is an Epstein Barr virus (EBV) associated malignant carcinoma. The EBV- positive NPC cells display much aggressiveness, which has been reported previously by various labs. It was reported that in papillomavirus type 16 E6- and E7- expressing keratinocytes, a high expression of UBE2C was observed, which may lead to the bypass of the spindle assembly checkpoint even with the DNA injury [40]. In NPC cells, EBV may impair cell cycle checkpoint via its encoded lament membrane protein [41]. Thus, the possible relationship between the infection of EBV and up-regulation of UBE2C in NPC should deserver much attention.

\section{Conclusions}

We provided the first evidence that high UBE2C expression is closely related to the clinical progression of NPC. UBE2C was universally expressed in all NPC cell lines examined, and its expression levels were inversely related with cell differentiation; knockdown of UBE2C by specific siRNA led to attenuated cell proliferation and cell cycle arrest at $\mathrm{G}_{2}-\mathrm{M}$ and $\mathrm{S}$ phases. Our results indicated that detection and targeting of UBE2C may be beneficial for NPC treatment.

\section{Competing interests}

The authors declare no competing interests.

\section{Authors' contributions}

ZS participated in the design of the study, performed statistical analysis and drafted the manuscript. XJ, ZC, RD performed the experiments. SZ, QH participated in the design of the study and helped to draft the manuscript. YZ collected the clinical samples and participated in the design of the study. $\mathrm{BL}, \mathrm{HJ}$ collected the clinical samples and scored the immunohistochemistry. JG, WJ conceived and coordinated the study. All the authors read and approved the final manuscript.

\section{Acknowledgments}

This work was supported by grants from the Doctoral Program of Guangdong Medical College (B2010013), the Sci-Tech Project Foundation of Zhanjiang City (2011C3107017), the National Natural Scientific Foundation of China (81060184, 81260350), and the Natural Foundation of Hainan Province of China (811201).

\section{Author details}

${ }^{1}$ Department of Pathology \& Pathophysiology, School of Basic Medicine Science, Guangdong Medical College, Zhanjiang 524023, People's Republic of China. ${ }^{2}$ Hainan Provincial Key Laboratory of Tropical Medicine, Hainan
Medical College, Haikou 571199, People's Republic of China. ${ }^{3}$ Department of Pathology, People's Hospital of Zhongshan City, Zhongshan 528400, People's Republic of China.

Received: 15 January 2013 Accepted: 12 April 2013

Published: 15 April 2013

\section{References}

1. Hershko A, Ciechanover A: The ubiquitin system. Annu Rev Biochem 1998 $67: 425-479$

2. Townsley FM, Aristarkhov A, Beck S, Hershko A, Ruderman JV: Dominantnegative cyclin-selective ubiquitin carrier protein $\mathrm{E} 2-\mathrm{C} / \mathrm{UbcH} 10$ blocks cells in metaphase. Proc Natl Acad Sci U S A 1997, 94:2362-2367.

3. Lin Y, Hwang WC, Basavappa R: Structural and functional analysis of the human mitotic-specific ubiquitin-conjugating enzyme, UbcH10. J Biol Chem 2002, 277:21913-21921.

4. Okamoto Y, Ozaki T, Miyazaki K, Aoyama M, Miyazaki M, Nakagawara A: UbcH10 is the cancer-related E2 ubiquitin-conjugating enzyme. Cancer Res 2003, 63:4167-4173.

5. Hao Z, Zhang H, Cowell J: Ubiquitin-conjugating enzyme UBE2C: molecular biology, role in tumorigenesis, and potential as a biomarker. Tumour Biol 2012, 33:723-730.

6. leta K, Ojima E, Tanaka F, Nakamura Y, Haraguchi N, Mimori K, Inoue H, Kuwano $\mathrm{H}$, Mori M: Identification of overexpressed genes in hepatocellular carcinoma, with special reference to ubiquitinconjugating enzyme E2C gene expression. Int J Cancer 2007, 121:33-38.

7. Pallante P, Berlingieri MT, Troncone G, Kruhoffer M, Orntoft TF, Viglietto G, Caleo A, Migliaccio I, Decaussin-Petrucci M, Santoro M, Palombini L, Fusco A: UbcH10 overexpression may represent a marker of anaplastic thyroid carcinomas. Br J Cancer 2005, 93:464-471.

8. Berlingieri MT, Pallante P, Sboner A, Barbareschi M, Bianco M, Ferraro A, Mansueto G, Borbone E, Guerriero E, Troncone G, Fusco A: UbcH10 is overexpressed in malignant breast carcinomas. Eur J Cancer 2007, 43:2729-2735

9. Fujita $T$, Ikeda $H$, Taira $N$, Hatoh $S$, Naito M, Doihara $H$ : Overexpression of UbcH10 alternates the cell cycle profile and accelerate the tumor proliferation in colon cancer. BMC Cancer 2009, 9:87.

10. Chen S, Chen Y, Hu C, Jing H, Cao Y, Liu X: Association of clinicopathological features with $\mathrm{UbcH} 10$ expression in colorectal cancer. J Cancer Res Clin Oncol 2010, 136:419-426.

11. Bose MV, Gopal G, Selvaluxmy G, Rajkumar T: Dominant negative Ubiquitin-conjugating enzyme E2C sensitizes cervical cancer cells to radiation. Int J Radiat Biol 2012, 88:629-634

12. Perrotta I, Bruno L, Maltese L, Russo E, Donato A, Donato G: Immunohistochemical analysis of the ubiquitin-conjugating enzyme UbcH10 in lung cancer: a useful tool for diagnosis and therapy. $J$ Histochem Cytochem 2012, 60:359-365.

13. Jiang L, Huang CG, Lu YC, Luo C, Hu GH, Liu HM, Chen JX, Han HX: Expression of ubiquitin-conjugating enzyme $\mathrm{E} 2 \mathrm{C} / \mathrm{UbcH} 10$ in astrocytic tumors. Brain Res 2008, 1201:161-166.

14. Chen SM, Jiang CY, Wu JY, Liu B, Chen YJ, Hu CJ, Liu XX: RNA interferencemediated silencing of UBCH10 gene inhibits colorectal cancer cell growth in vitro and in vivo. Clin Exp Pharmacol Physiol 2010, 37:525-529.

15. Jiang L, Bao Y, Luo C, Hu G, Huang C, Ding X, Sun K, Lu Y: Knockdown of ubiquitin-conjugating enzyme $\mathrm{E} 2 \mathrm{C} / \mathrm{UbcH} 10$ expression by RNA interference inhibits glioma cell proliferation and enhances cell apoptosis in vitro. J Cancer Res Clin Oncol 2010, 136:211-217.

16. van Ree $\mathrm{JH}$, Jeganathan KB, Malureanu L, van Deursen JM: Overexpression of the E2 ubiquitin-conjugating enzyme $\mathrm{UbcH} 10$ causes chromosome missegregation and tumor formation. J Cell Biol 2010, 188:83-100.

17. Cao SM, Simons MJ, Qian CN: The prevalence and prevention of nasopharyngeal carcinoma in China. Chin J Cancer 2011, 30:114-119.

18. Ho FC, Tham IW, Earnest A, Lee KM, Lu JJ: Patterns of regional lymph node metastasis of nasopharyngeal carcinoma: a meta-analysis of clinical evidence. BMC Cancer 2012, 12:98.

19. Cho WC: Nasopharyngeal carcinoma: molecular biomarker discovery and progress. Mol Cancer 2007, 6:1

20. Donato G, lofrida G, Lavano A, Volpentesta G, Signorelli F, Pallante PL, Berlingieri MT, Pierantoni MG, Palmieri D, Conforti F, Maltese L, Tucci L, Amorosi A, Fusco A: Analysis of UbcH10 expression represents a useful 
tool for the diagnosis and therapy of astrocytic tumors. Clin Neuropathol 2008, 27:219-223.

21. Jie W, He QY, Luo BT, Zheng SJ, Kong YQ, Jiang HG, Li RJ, Guo JL, Shen ZH Inhibition of Pim-1 attenuates the proliferation and migration in nasopharyngeal carcinoma cells. Asian Pac J Trop Med 2012, 5:645-650.

22. Fang W, Li X, Jiang Q, Liu Z, Yang H, Wang S, Xie S, Liu Q, Liu T, Huang J, Xie W, Li Z, Zhao Y, Wang E, Marincola FM, Yao K: Transcriptional patterns, biomarkers and pathways characterizing nasopharyngeal carcinoma of Southern China. J Transl Med 2008, 6:32

23. Takahashi $Y$, Ishii $Y$, Nishida $Y$, Ikarashi M, Nagata T, Nakamura T, Yamamori $S$, Asai S: Detection of aberrations of ubiquitin-conjugating enzyme E2C gene (UBE2C) in advanced colon cancer with liver metastases by DNA microarray and two-color FISH. Cancer Genet Cytogenet 2006, 168:30-35.

24. Berlingieri MT, Pallante $P$, Guida M, Nappi C, Masciullo V, Scambia G, Ferraro A, Leone V, Sboner A, Barbareschi M, Ferro A, Troncone G, Fusco A: UbcH10 expression may be a useful tool in the prognosis of ovarian carcinomas. Oncogene 2007, 26:2136-2140.

25. Lin J, Raoof DA, Wang Z, Lin MY, Thomas DG, Greenson JK, Giordano TJ, Orringer MB, Chang AC, Beer DG, Lin L: Expression and effect of inhibition of the ubiquitin-conjugating enzyme $\mathrm{E} 2 \mathrm{C}$ on esophageal adenocarcinoma. Neoplasia 2006, 8:1062-1071.

26. Rajkumar T, Sabitha K, Vijayalakshmi N, Shirley S, Bose MV, Gopal G, Selvaluxmy G: Identification and validation of genes involved in cervical tumourigenesis. BMC Cancer 2011, 11:80.

27. Troncone G, Guerriero E, Pallante P, Berlingieri MT, Ferraro A, Del Vecchio L, Gorrese M, Mariotti E, laccarino A, Palmieri EA, Zeppa P, Palombini L, Fusco A: UbcH10 expression in human lymphomas. Histopathology 2009, 54:731-740.

28. Psyrri A, Kalogeras KT, Kronenwett R, Wirtz RM, Batistatou A, Bournakis E, Timotheadou E, Gogas H, Aravantinos G, Christodoulou C, Makatsoris T, Linardou H, Pectasides D, Pavlidis N, Economopoulos T, Fountzilas G: Prognostic significance of UBE2C mRNA expression in high-risk early breast cancer. A Hellenic Cooperative Oncology Group (HeCOG) study. Ann Oncol 2012, 23:1422-1427.

29. Vasiljevic A, Champier J, Figarella-Branger D, Wierinckx A, Jouvet A, FevreMontange M: Molecular characterization of central neurocytomas: potential markers for tumor typing and progression. Neuropathology 2013, 33:149-161.

30. Bavi P, Uddin S, Ahmed M, Jehan Z, Bu R, Abubaker J, Sultana M, Al-Sanea N, Abduljabbar A, Ashari LH, Alhomoud S, Al-Dayel F, Prabhakaran S, Hussain AR, Al-Kuraya KS: Bortezomib stabilizes mitotic cyclins and prevents cell cycle progression via inhibition of UBE2C in colorectal carcinoma. Am J Pathol 2011, 178:2109-2120.

31. Wagner KW, Sapinoso LM, El-Rifai W, Frierson HF, Butz N, Mestan J, Hofmann F, Deveraux QL, Hampton GM: Overexpression, genomic amplification and therapeutic potential of inhibiting the $\mathrm{UbcH} 10$ ubiquitin conjugase in human carcinomas of diverse anatomic origin. Oncogene 2004, 23:6621-6629.

32. Wang H, Zhang $C$, Rorick A, Wu D, Chiu M, Thomas-Ahner J, Chen Z, Chen H, Clinton SK, Chan KK, Wang Q: CCI-779 inhibits cell-cycle G2-M progression and invasion of castration-resistant prostate cancer via attenuation of UBE2C transcription and mRNA stability. Cancer Res 2011, 71:4866-4876

33. Lee JJ, Au AY, Foukakis T, Barbaro M, Kiss N, Clifton-Bligh R, Staaf J, Borg A, Delbridge L, Robinson BG, Wallin G, Hoog A, Larsson C: Array-CGH identifies cyclin D1 and UBCH10 amplicons in anaplastic thyroid carcinoma. Endocr Relat Cancer 2008, 15:801-815.

34. Tzelepi V, Zhang J, Lu JF, Kleb B, Wu G, Wan X, Hoang A, Efstathiou E, Sircar K, Navone NM, Troncoso P, Liang S, Logothetis CJ, Maity SN, Aparicio AM: Modeling a lethal prostate cancer variant with small-cell carcinoma features. Clin Cancer Res 2012, 18:666-677.

35. Lo KW, Chung GT, To KF: Deciphering the molecular genetic basis of NPC through molecular, cytogenetic, and epigenetic approaches. Semin Cancer Biol 2012, 22:79-86.

36. Li X, Wang E, Zhao YD, Ren JQ, Jin P, Yao KT, Marincola FM: Chromosomal imbalances in nasopharyngeal carcinoma: a meta-analysis of comparative genomic hybridization results. J Trans/ Med 2006, 4:4.

37. Lo KW, Teo PM, Hui AB, To KF, Tsang YS, Chan SY, Mak KF, Lee JC, Huang DP: High resolution allelotype of microdissected primary nasopharyngeal carcinoma. Cancer Res 2000, 60:3348-3353.
38. Hu C, Wei W, Chen X, Woodman CB, Yao Y, Nicholls JM, Joab I, Sihota SK, Shao JY, Derkaoui KD, Amari A, Maloney SL, Bell Al, Murray PG, Dawson CW, Young LS, Arrand JR: A global view of the oncogenic landscape in nasopharyngeal carcinoma: an integrated analysis at the genetic and expression levels. PLoS One 2012, 7:e41055.

39. Yan W, Song L, Wei W, Li A, Liu J, Fang Y: Chromosomal abnormalities associated with neck nodal metastasis in nasopharyngeal carcinoma. Tumour Biol 2005, 26:306-312.

40. Patle D, McCance DJ: Compromised spindle assembly checkpoint due to altered expression of Ubch10 and Cdc20 in human papillomavirus type 16 E6- and E7-expressing keratinocytes. Virol 2010, 84:10956-10964.

41. Deng W, Pang PS, Tsang CM, Hau PM, Yip YL, Cheung AL, Tsao SW: EpsteinBarr virus-encoded latent membrane protein 1 impairs $G 2$ checkpoint in human nasopharyngeal epithelial cells through defective Chk1 activation. PLoS One 2012, 7:e39095.

doi:10.1186/1471-2407-13-192

Cite this article as: Shen et al:: High expression of ubiquitin-conjugating enzyme 2C (UBE2C) correlates with nasopharyngeal carcinoma progression. BMC Cancer 2013 13:192.

\section{Submit your next manuscript to BioMed Central and take full advantage of:}

- Convenient online submission

- Thorough peer review

- No space constraints or color figure charges

- Immediate publication on acceptance

- Inclusion in PubMed, CAS, Scopus and Google Scholar

- Research which is freely available for redistribution 